Pacific Journal of Mathematics

PRODUCTS OF POSITIVE REFLECTIONS IN REAL 


\title{
PRODUCTS OF POSITIVE REFLECTIONS IN REAL ORTHOGONAL GROUPS
}

\author{
DRAGOMIR Ž. DJOKOVIĆ
}

\begin{abstract}
Let $O(f)$ be the orthogonal group of a symmetric bilinear form $f$ defined on a finite-dimensional real vector space $V$. If $f$ is indefinite then $O(f)$ has two conjugacy classes of reflections, one of which consists of so called positive reflections. We denote by $G^{+}$the subgroup of $O(f)$ generated by all positive reflections. In this paper we describe this subgroup and solve the length problem in $G^{+}$with respect to the distinguished set of generators. When $f$ is non-degenerate this problem was solved by $\mathbf{J}$. Malzan. Our proof (in the case of arbitrary $f$ ) is shorter and completely different from his proof.
\end{abstract}

Introduction. Let $O(f)$ be the orthogonal group of a symmetric bilinear form $f$ defined on a finite-dimensional real vector space $V$. If $f$ is indefinite then $O(f)$ has two conjugacy classes of reflections, one of which consists of so called positive reflections. We denote by $G^{+}$the subgroup of $O(f)$ generated by all positive reflections. In this paper we solve the length problem in $G^{+}$with respect to the distinguished set of generators. When $f$ is non-degenerate this problem was solved by $\mathbf{J}$. Malzan. Our prooof (in the case of arbitrary $f$ ) is shorter and completely different from his proof.

A non-isotropic vector $a$ determines a unique orthogonal reflection $R_{a}$ and we say that $R_{a}$ is positive if $f(a, a)>0$. The weak orthogonal group $O^{*}(f)$ consists of all isometries which fix every vector in $\operatorname{Rad} V$. To avoid trivial and known cases let us assume that $f$ is indefinite, i.e., that $f(x, x)$ takes both positive and negative values. Then $O^{*}(f) \supset G^{+} \supset O_{1}^{*}(f)$ where $O_{1}^{*}(f)$ denotes the identity component of $O^{*}(f)$. Moreover $O^{*}(f) / O_{1}^{*}(f) \cong Z_{2} \times Z_{2}$ and $G^{+} / O_{1}^{*}(f) \cong Z_{2}$.

Our main theorem (Theorem 2) gives explicit formulas for the length of any $u \in G^{+}$with respect to the generating set consisting of all positive reflections. When $f$ is nondegenerate this result is due to J. Malzan [5]. The proof is based on some earlier results of M. Götzky [3] on $O^{*}(f)$. One should point out that Götzky considers also weak unitary groups and his underlying field $F$ is arbitrary (char $F \neq 2$ in the case of $O^{*}(f)$ ).

The main idea of the proof is to take a shortest representation of $u \in G^{+}$as a product of reflections and then try to convert all reflections 
into positive ones. This method is effective in the generic case; the exceptional cases are treated separately.

1. Weak orthogonal groups in general. Let $V$ be a finite-dimensional vector space over a field $F$, $\operatorname{char} F \neq 2$, and let $f$ be a symmetric bilinear form on $V$. An automorphism $u$ of $V$ is called an isometry if $f(u(x), u(y))=f(x, y)$ for all $x, y \in V$. The group of all isometries will be denoted by $O(f)$ and we refer to it as the orthogonal group of the form $f$. (Note that we allow $f$ to be degenerate.)

The weak orthogonal group $O^{*}(f)$ is the subgroup of $O(f)$ consisting of all isometries which fix every vector in the radical $\operatorname{Rad} V=\{x \in V$ : $f(x, y)=0, \forall y \in V\}$.

For $u \in O(f)$ we define its fixed space Fix $u$ and its residual space Res $u$ by

$$
\text { Fix } u=\operatorname{Ker}(u-1), \quad \operatorname{Res} u=\operatorname{Im}(u-1) .
$$

We also define the residue $r(u)$ and the radical residue $r_{0}(u)$ of $u$ to be

$$
r(u)=\operatorname{dim} \operatorname{Res} u, \quad r_{0}(u)=\operatorname{dim}(\operatorname{Res} u \cap \operatorname{Rad} V) .
$$

If $a$ is a non-isotropic vector, i.e., $f(a, a) \neq 0$, then the transformation $R_{a}: V \rightarrow V$ defined by

$$
R_{a}(x)=x-2 f(a, x) f(a, a)^{-1} a
$$

belongs to $O^{*}(f)$ and is called a reflection. We have

$$
\text { Fix } R_{a}=\langle a\rangle^{\perp}, \quad \operatorname{Res} R_{a}=\langle a\rangle
$$

and $R_{a}(a)=-a$. (For any subspace $W$ of $V$ we denote by $W^{\perp}$ the orthogonal complement of $W$ with respect to the form $f$.)

We shall now state some results of M. Götzky [3] concerning the group $O^{*}(f)$. (In his paper he also treats the weak unitary groups but we shall not need those results.) For further results and generalizations we refer the reader to a paper of E. Ellers [2].

Every $u \in O^{*}(f)$ can be expressed as a product of reflections

$$
u=R_{a_{1}} R_{a_{2}} \cdots R_{a_{m}} .
$$

Since det $R_{a}=-1$ for every reflection $R_{a}$, it follows that det $u= \pm 1$ for all $u \in O^{*}(f)$. Moreover the subgroup

$$
S O^{*}(f)=\left\{u \in O^{*}(f): \operatorname{det} u=1\right\}
$$

has index 2 in $O^{*}(f)$. 
For $u \in O^{*}(f)$ we shall denote by $l(u)$ the length of $u$ with respect to the generating set consisting of all reflections. Thus $l(u)$ is the smallest integer $m(\geq 0)$ for which a factorization (1) exists.

TheOREM 1. (M. Götzky) For $u \in O^{*}(f)$ we have $l(u)=r(u)+r_{0}(u)$ except when (Fix $u)^{\perp}$ is totally isotropic and $u \neq 1$. In the exceptional case we have $l(u)=r(u)+r_{0}(u)+2$.

When $f$ is non-degenerate, i.e., $\operatorname{Rad} V=0$; this theorem is due to $\mathrm{P}$. Scherk [6].

2. Real case and the statement of the main result. From now on we shall assume that $F$ is the real field $R$. A vector $x$ is called positive (resp. negative) if $f(x, x)>0$ (resp. $f(x, x)<0$ ). We shall denote by $n$ the dimension of $V$ and by $(p, q, s)$ the signature of $f$. This means that every orthogonal basis of $V$ consists of $p$ positive vectors, $q$ negative vectors, and $s$ isotropic vectors.

A reflection $R_{a}$ is positive (resp. negative) if $a$ is positive (resp. negative). It follows from Witt's theorem that all positive (resp. negative) reflections are conjugate in $O^{*}(f)$. We shall denote by $G^{+}$(resp. $G^{-}$) the subgroup of $O^{*}(f)$ generated by all positive (resp. negative) reflections. If $p=0$, i.e., $f$ is negative semidefinite then there are no positive reflections and we have $G^{+}=\{1\}$ and $G^{-}=O^{*}(f)$. If $q=0$ then $G^{+}=O^{*}(f)$ and $G^{-}=\{1\}$.

In view of these remarks and Theorem 1 we shall assume throughout that $f$ is indefinite, i.e., $p \geq 1$ and $q \geq 1$. Clearly $O(f)$ and $O^{*}(f)$ are real algebraic groups and so Lie groups. Let $O_{1}^{*}(f)$ be the identity component of $O^{*}(f)$ viewed as a Lie group.

Let $V=V_{1} \oplus \operatorname{Rad} V$ and let $f_{1}$ be the restriction of $f$ to $V_{1} \times V_{1}$. Clearly $f_{1}$ is a non-degenerate symmetric bilinear form on $V_{1}$ of signature $(p, q, 0)$. Then the elements $u$ of $O(f)$ are represented by matrices

$$
u=\left(\begin{array}{cc}
u_{1} & 0 \\
v & u_{0}
\end{array}\right)
$$

where $u_{1} \in O\left(f_{1}\right), u_{0}$ is an automorphism of $\operatorname{Rad} V$ and $v: V_{1} \rightarrow \operatorname{Rad} V$ is an arbitrary linear map. We have $u \in O^{*}(f)$ if and only if $u_{0}=1$.

LEMMA 1. $O^{*}(f) / O_{1}^{*}(f) \cong Z_{2} \times Z_{2}$. 
Proof. If $s=0$ this is well known, see e.g. [4, Lemma 2.4(b), p. 451]. In general the assertion follows from this special case and the above matrix description of elements of $O^{*}(f)$.

COROLlaRY. $G^{+} \cdot O_{1}^{*}(f) / O_{1}^{*}(f)$ and $G^{-} \cdot O_{1}^{*}(f) / O_{1}^{*}(f)$ are cyclic groups of order two. The three subgroups $G^{+} O_{1}^{*}(f), G^{-} \cdot O_{1}^{*}(f)$, and $S O^{*}(f)$ are distinct.

Proof. Since all positive (resp. negative) reflections are conjugate in $O^{*}(f)$, they lie in a single connected component of $O^{*}(f)$. This implies the first assertion. We have $G^{+} O_{1}^{*}(f) \neq G^{-} O_{1}^{*}(f)$ because $O^{*}(f)$ is generated by reflections. These two groups are different from $S O^{*}(f)$ because det $R=-1$ for each reflection $R$.

For $u \in G^{+}$we shall denote by $l^{+}(u)$ the length of $u$ with respect to the generating set consisting of all positive reflections. We can now state our main result.

THEOREM 2. We have $G^{+} \supset O_{1}^{*}(f)$. For $u \in G^{+}$we have $l^{+}(u)=$ $r(u)+r_{0}(u)$ except in the following cases:

(i) The subspace (Fix $u)^{\perp}$ is negative semidefinite and $u \neq 1$,

(ii) $u^{2}=1$ and $u(x)=-x$ for some negative vector $x$.

In the exceptional cases we have $l^{+}(u)=r(u)+r_{0}(u)+2$.

When $f$ is non-degenerate this theorem is due to J. Malzan [5]. Our proof below even in the more general case is simpler and more elementary than his. For instance we do not need the detailed knowledge of the conjugacy classes of $O(f)$, which is heavily used in [5] in the case when $f$ is non-degenerate.

3. Proofs. We shall assume that the reader is familiar with Götzky's paper [3] and we shall use some of his technical lemmas in addition to Theorem 1. The main tool in our proof is the following technical lemma.

LEMMA 2. Let $a, b, c$ be linearly independent vectors with a positive and $b$ and $c$ negative. If the sequence $a, b, c$ is not orthogonal then the isometry $u=R_{a} R_{b} R_{c}$ can be written as a product of three positive reflections.

Proof. Without any loss of generality we may assume that $f(a, a)=1$ and $f(b, b)=f(c, c)=-1$. Set $f(a, b)=\alpha, f(a, c)=\beta$, and $f(b, c)=\gamma$. By hypothesis at least one of $\alpha, \beta, \gamma$ is non-zero. Since $R_{b} R_{c}=R_{d} R_{b}$ 
where $d=R_{b}(c)$, we may assume that in fact $\beta$ or $\gamma$ is non-zero. Then for $e=(\eta-\alpha \xi) a+\xi b$ we have

$$
f(e, e)=(\eta-\alpha \xi)^{2}-\xi^{2}+2 \alpha \xi(\eta-\alpha \xi)=\eta^{2}-\left(1+\alpha^{2}\right) \xi^{2},
$$

and

$$
\Delta=\left|\begin{array}{ll}
f(c, c) & f(c, e) \\
f(e, c) & f(e, e)
\end{array}\right|=\left(1+\alpha^{2}\right) \xi^{2}-\eta^{2}-(\beta \eta+(\gamma-\alpha \beta) \xi)^{2} .
$$

Since $\beta$ or $\gamma$ is not zero, we can choose $\xi$ and $\eta$ so that $f(e, e)=-1$ and $\Delta<0$. By Dreispiegelungssatz [1, Proposition 6.1] the product $R=$ $R_{a} R_{b} R_{e}$ is a reflection. Since $b$ and $e$ are negative vectors, we have $R_{b} R_{e} \in O_{1}^{*}(f)$ and so $R$ must be a positive reflection by Lemma 1, Cor. We have $u=R R_{e} R_{c}$ where $R_{e}$ and $R_{c}$ are negative reflections. Since $\Delta<0$ the space $W=\langle c, e\rangle$ is a hyperbolic plane. We claim that $R_{e} R_{c}$ is a product of two positive reflections. To prove this it suffices to consider the restrictions of $R_{e}$ and $R_{c}$ to $W$. Then in $W$ the operators $-R_{e}$ and $-R_{c}$ are positive reflections whose product is $R_{e} R_{c}$. This completes the proof.

Proof of Theorem 2. Let $u \in G^{+} \cdot O_{1}^{*}(f)$.

Case 1. $u$ is not exceptional, i.e., neither (i) nor (ii) holds.

Clearly $l^{+}(u) \geq l(u)$ and by Theorem $1, l(u)=r(u)+r_{0}(u)$. Write $m=l(u)$ and let (1) be a factorization of $u$ into a product of $m$ reflections containing a maximal number, say $k$, of positive reflections. We have to prove that $k=m$.

This is clear if $m=0$, i.e., $u=1$. Otherwise we prove first that $k \geq 1$. Since (i) does not hold there exists a positive vector $a \in(\text { Fix } u)^{\perp}$. It follows from [3, Hilfssatz 2.1, p. 385] that for $v=R_{a} u$ we have $r(v)=r(u)$ and $r_{0}(v)=r_{0}(u)-1$. By Theorem $1 l(v)=m-1$ and since $u=R_{a} v$ we have $k \geq 1$. We may assume that the vectors $a_{i}$ are positive for $1 \leq i \leq k$ and negative for $k<i \leq m$.

Now assume that $k<m$. By Lemma 1 , Cor. $m-k$ must be even, and so $k \leq m-2$. Assume that for every pair of indices $(i, j)$ such that $1 \leq i<j \leq m$ and $j>k$ we have $a_{i} \perp a_{j}$. Since (ii) does not hold there must exist a pair of indices $(i, j)$ such that $1 \leq i<j \leq k$ and $f\left(a_{i}, a_{j}\right) \neq 0$. Without any loss of generality we may assume that $f\left(a_{k-1}, a_{k}\right) \neq 0$. Let 
$b \in\left\langle a_{k}, a_{k+1}\right\rangle$ be a positive vector such that $b \notin\left\langle a_{k}\right\rangle$. By Dreispiegelungssatz the product $R_{b} R_{a_{k}} R_{a_{k+1}}$ is a reflection, say $R_{c}$, and by Lemma 1, Cor. it is a negative reflection. Thus we can replace in (1) the product $R_{a_{k}} R_{a_{k+1}}$ by $R_{b} R_{c}$. Note that $f\left(a_{k-1}, c\right) \neq 0$. This shows that we may assume that there exists a pair of indices $(i, j)$ such that $1 \leq i<j \leq m$, $j>k$ and $f\left(a_{i}, a_{j}\right) \neq 0$. Without any loss of generality we may in fact assume that the sequence $a_{k}, a_{k+1}, a_{k+2}$ is not orthogonal. By Lemma 2 the product $R_{a_{k}} R_{a_{k+1}} R_{a_{k+2}}$ can be replaced by a product of three positive reflections. This contradicts the maximality of $k$.

Hence we have shown that $k=m$, and in particular $u \in G^{+}$.

Case 2. (i) or (ii) holds. Let $m=r(u)+r_{0}(u)$. We prove first that $l^{+}(u) \geq m+2$. This is clear if $l(u)=m+2$. Otherwise we have $l(u)=m$ and since det $u=(-1)^{m}$, it suffices to show that $u$ cannot be written as a product of $m$ positive reflections. Assume that it can and let (1) be such a factorization.

We claim that $a_{k} \in(\operatorname{Fix} u)^{\perp}$ for all $k$. It suffices to prove this for $k=1$. Thus let us assume that $a_{1} \notin(\text { Fix } u)^{\perp}$. Then by [3, Proposition 2.1.3] for $v=R_{a_{1}} u$ we have $\operatorname{Res} v=\operatorname{Res} u \oplus\left\langle a_{1}\right\rangle$, and consequently $r(v)=r(u)+1$ and $r_{0}(v)=r_{0}(u)$. It follows that

$$
l(v)=r(v)+r_{0}(v)=r(u)+r_{0}(u)+1=m+1 .
$$

This is a contradiction since $v$ is a product of $m-1$ reflections. Hence our claim is proved.

If (i) holds then since $a_{k} \in(\text { Fix } u)^{\perp}$ for all $k$, we conclude that all reflections in (1) are negative, contrary to our hypothesis. Thus if (i) holds then $l^{+}(u) \geq m+2$.

Now assume that (ii) holds. Since $u^{2}=1$ we have $V=$ Fix $u \oplus \operatorname{Res} u$ and Fix $u \perp \operatorname{Res} u$. Since $\operatorname{Rad} V \subset$ Fix $u$, it follows that $\operatorname{Res} u$ is non-degenerate, $r_{0}(u)=0$, and so $m=r(u)$. From (1) it follows that $\operatorname{Res} u \subset$ $\left\langle a_{1}, \ldots, a_{m}\right\rangle$, see e.g. $[2, \S 3]$. Since $r(u)=m$, we conclude that $a_{1}, \ldots, a_{m}$ is a basis of Res $u$.

We claim that this basis is orthogonal. It suffices to show that $a_{1} \perp a_{i}$ for $2 \leq i \leq m$. Let $b$ be a non-zero vector in Res $u$ such that $b \perp a_{i}$ for $2 \leq i \leq m$. Since $u$ is -1 on $\operatorname{Res} u$, we have $u(b)=-b$. On the other hand it follows from (1) that $u(b)=R_{a_{1}}(b)$. Hence we have $R_{a_{1}}(b)=-b$ and so $a_{1} \in\langle b\rangle$. This proves our claim.

Since the basis $a_{1}, \ldots, a_{m}$ of Res $u$ is orthogonal and each of these vectors is positive, we conclude that $\operatorname{Res} u$ is a positive definite subspace. 
This contradicts (ii). Hence also in the case (ii) we must have $l^{+}(u) \geq$ $m+2$.

It remains to show that $l^{+}(u) \leq m+2$, i.e., that $u$ can be written as a product of $m+2$ positive reflections.

Assume first that (i) holds. Since the positive vectors form an open set in $V$, we can choose a positive vector $a$ such that $a \notin$ Fix $u$. Since (i) holds we have also $a \notin(\text { Fix } u)^{\perp}$. Therefore Fix $u$ is not invariant under $R_{a}$. Hence we can choose $x \in$ Fix $u$ such that $R_{a}(x) \notin$ Fix $u$. Let $v=R_{a} u$ and note that

$$
v^{2}(x)=R_{a} u R_{a}(x) \neq R_{a} R_{a}(x)=x,
$$

and so $v^{2} \neq 1$. By [3, Proposition 2.1.3] we have $\operatorname{Res} v=\operatorname{Res} u \oplus\langle a\rangle$, and so $r(v)=r(u)+1$ and $r_{0}(v)=r_{0}(u)$. Thus $v$ is non-exceptional and by the result of Case 1 we have

$$
l^{+}(v)=l(v)=r(v)+r_{0}(v)=m+1 .
$$

Since $u=R_{a} v, u$ is a product of $m+2$ positive reflections.

Now assume that (ii) holds. Choose an orthogonal basis $a_{1}, \ldots, a_{m}$ of Res $u$ such that $a_{1}, \ldots, a_{k}$ are positive and $a_{k+1}, \ldots, a_{m}$ are negative vectors. It follows from (ii) that $k<m$. Let

$$
v=R_{a_{1}} \cdots R_{a_{k}} u .
$$

This $v$ satisfies (i) and we have $l(v)=m-k$. Hence $l^{+}(v)=m-k+2$ by the result just proved above, and so $l^{+}(u) \leq m+2$.

This completes the proof of Theorem 2.

REMARK. It is easy to modify Theorem 2 so that it applies to the case when $V$ is infinite-dimensional. Clearly if $u \in G^{+}$then $r(u)<\infty$. The length formulas of Theorem 2 remain valid.

\section{REFERENCES}

[1] J. Ahrens, A. Dress, and H. Wolff, Relationen zwischen Symmetrien in orthogonalen Gruppen, J. fur Reine und Angew. Math., 234 (1969), 1-11.

[2] E. W. Ellers, Decomposition of orthogonal, symplectic and unitary isometries into simple isometries, Abh. Math. Sem. Univ. Hamburg, 46 (1977), 97-127.

[3] M. Götzky, Über die Erzeugenden der engeren unitären Gruppen, Arch. der Math., 19 (1968), 383-389.

[4] S. Helgason, Differential geometry, Lie groups, and symmetric spaces, Academic Press, New York, 1978. 
[5] J. Malzan, Products of positive reflections in the orthogonal group, Canad. J. Math., 34 (1982), 484-499.

[6] P. Scherk, On the decomposition of orthogonalities into symmetries, Proc. Amer. Math. Soc., 1 (1950), 481-491.

Received September 10, 1981.

Department of PURe Mathematics

UNIVERSITY OF WATERLOO

WATERLOO, ONTARIO

CANADA N2L 3G1 


\title{
PACIFIC JOURNAL OF MATHEMATICS \\ EDITORS
}

\author{
DONALD BABBITT (Managing Editor) \\ University of California \\ Los Angeles, CA 90024 \\ Hugo Rossi \\ University of Utah \\ Salt Lake City, UT 84112 \\ C. C. Moore and Arthur Ogus \\ University of California \\ Berkeley, CA 94720
}

\author{
J. DugundiI \\ Department of Mathematics \\ University of Southern California \\ Los Angeles, CA 90089-1113
}

R. FINN and H. SAMELSON

Stanford University

Stanford, CA 94305

\begin{tabular}{lllll}
\multicolumn{3}{c}{ ASSOCIATE EDITORS } \\
R. ARENS & $\begin{array}{lll}\text { E. F. BeCKendach } \\
(1906-1982)\end{array}$ & B. H. NeumanN & F. Wolf & K. Yoshida
\end{tabular}

\begin{tabular}{ll}
\multicolumn{2}{c}{ SUPPORTING INSTITUTIONS } \\
UNIVERSITY OF ARIZONA & UNIVERSITY OF OREGON \\
UNIVERSITY OF BRITISH COLUMBIA & UNIVERSITY OF SOUTHERN CALIFORNIA \\
CALIFORNIA INSTITUTE OF TECHNOLOGY & STANFORD UNIVERSITY \\
UNIVERSITY OF CALIFORNIA & UNIVERSITY OF HAWAII \\
MONTANA STATE UNIVERSITY & UNIVERSITY OF TOKYO \\
UNIVERSITY OF NEVADA, RENO & UNIVERSITY OF UTAH \\
NEW MEXICO STATE UNIVERSITY & WASHINGTON STATE UNIVERSITY \\
OREGON STATE UNIVERSITY & UNIVERSITY OF WASHINGTON
\end{tabular}

The Supporting Institutions listed above contribute to the cost of publication of this Journal, but they are not owners or publishers and have no responsibility for its content or policies.

Mathematical papers intended for publication in the Pacific Journal of Mathematics should be in typed form or offset-reproduced (not dittoed), double spaced with large margins. Please do not use built up fractions in the text of the manuscript. However, you may use them in the displayed equations. Underline Greek letters in red, German in green, and script in blue. The first paragraph must be capable of being used separately as a synopsis of the entire paper. In particular it should contain no bibliographic references. Please propose a heading for the odd numbered pages of less than 35 characters. Manuscripts, in triplicate, may be sent to any one of the editors. Please classify according to the scheme of Math. Reviews, Index to Vol. 39. Supply name and address of author to whom proofs should be sent. All other communications should be addressed to the managing editor, or Elaine Barth, University of California, Los Angeles, California 90024.

There are page-charges associated with articles appearing in the Pacific Journal of Mathematics. These charges are expected to be paid by the author's University, Government Agency or Company. If the author or authors do not have access to such Institutional support these charges are waived. Single authors will receive 50 free reprints; joint authors will receive a total of 100 free reprints. Additional copies may be obtained at cost in multiples of 50 .

The Pacific Journal of Mathematics is issued monthly as of January 1966. Regular subscription rate: $\$ 132.00$ a year (6 Vol., 12 issues). Special rate: $\$ 66.00$ a year to individual members of supporting institutions.

Subscriptions, orders for numbers issued in the last three calendar years, and changes of address should be sent to Pacific Journal of Mathematics, P.O. Box 969, Carmel Valley, CA 93924, U.S.A. Old back numbers obtainable from Kraus Periodicals Co., Route 100, Millwood, NY 10546.

The Pacific Journal of Mathematics ISSN 0030-8730 is published monthly by the Pacific Journal of Mathematics at P.O. Box 969, Carmel Valley, CA 93924. Application to mail at Second-class postage rates is pending at Carmel Valley, California, and additional mailing offices. Postmaster: Send address changes to Pacific Journal of Mathematics, P. O. Box 969, Carmel Valley, CA 93924.

PUBLISHED BY PACIFIC JOURNAL OF MATHEMATICS, A NON-PROFIT CORPORATION

Copyright $\odot 1983$ by Pacific Journal of Mathematics 


\section{Pacific Journal of Mathematics}

Vol. 107, No. $2 \quad$ February, 1983

Driss Abouabdillah, Topologies de corps $A$ linéaires $\ldots \ldots \ldots \ldots \ldots . \ldots 257$

Patrick Robert Ahern, On the behavior near a torus of functions

holomorphic in the ball $\ldots \ldots \ldots \ldots \ldots \ldots \ldots \ldots \ldots \ldots \ldots \ldots \ldots . \ldots 267$

Donald Werner Anderson, There are no phantom cohomology operations

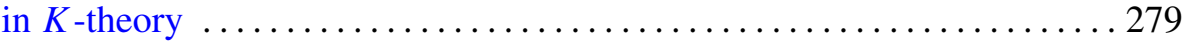

Peter Bloomfield, Nicolas P. Jewell and Eric Hayashi, Characterizations of

completely nondeterministic stochastic processes . ............. 307

Sydney Dennis Bulman-Fleming and K. McDowell, Absolutely flat

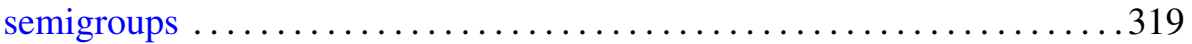

C. Debiève, On a Radon-Nikodým problem for vector-valued measures . . . 335

Dragomir Z. Djokovic, Products of positive reflections in real orthogonal

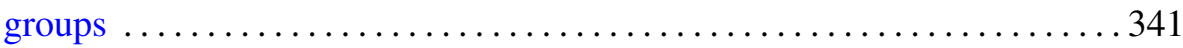

Thomas Farmer, The dual of the nilradical of the parabolic subgroups of

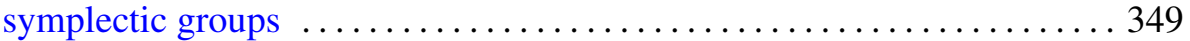

Gary R. Greenfield, Uniform distribution in subgroups of the Brauer group of an algebraic number field $\ldots \ldots \ldots \ldots \ldots \ldots \ldots \ldots \ldots \ldots \ldots . \ldots \ldots$

Paul Daniel Hill, When $\operatorname{Tor}(A, B)$ is a direct sum of cyclic groups $\ldots \ldots \ldots 383$

Hiroshi Maehara, Regular embeddings of a graph $\ldots \ldots \ldots \ldots \ldots \ldots \ldots 3$

Nikolaos S. Papageorgiou, Nonsmooth analysis on partially ordered vector spaces. I. Convex case . .............................4 403

Louis Jackson Ratliff, Jr., Powers of ideals in locally unmixed Noetherian

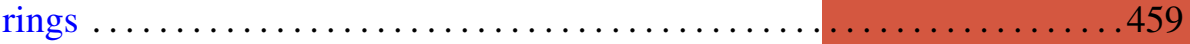

F. Dennis Sentilles and Robert Francis Wheeler, Pettis integration via the Stonian transform .......................... 